Cold Spring Harbor Perspect Biol 3: a007526 (2011)

\title{
Protein Folding and Quality Control in the ER
}

\section{Kazutaka Araki and Kazuhiro Nagata}

In the original version of this article, some of the text labels in Figure $2 \mathrm{C}$ were incorrect.

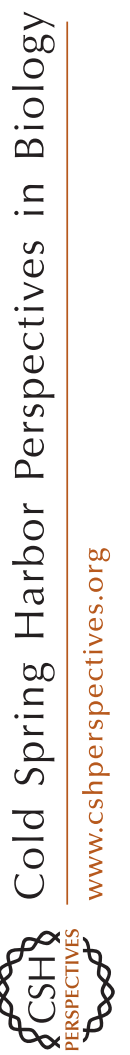

The publisher apologizes for these errors. The correct Figure 2 is reprinted below, and the PDF and HTML versions of the article have been corrected accordingly. 
K. Araki and K. Nagata
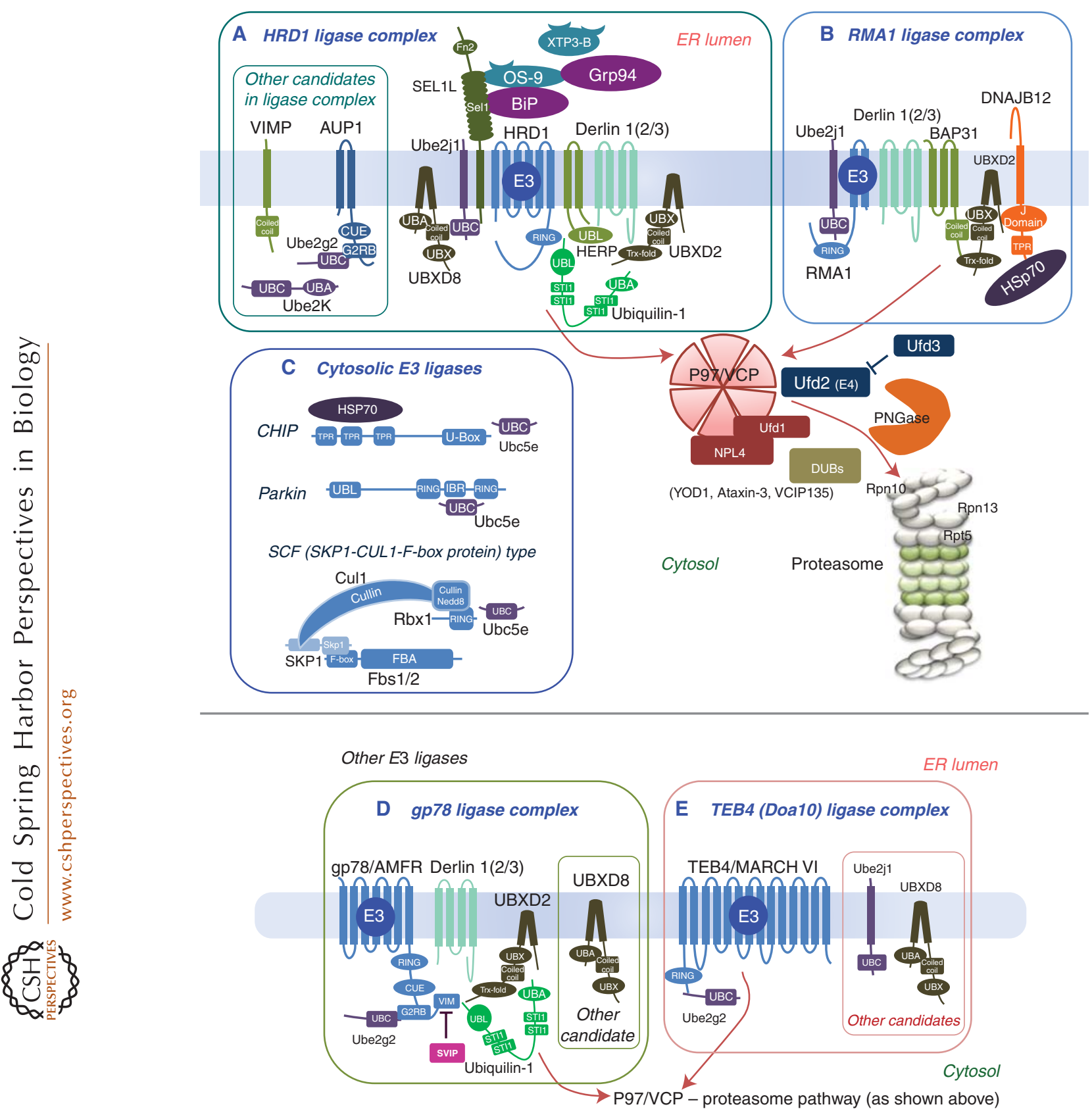

Figure 2. Schematic models of mammalian E3 ligase complexes. Selected components are depicted in each model (Tsai and Weissman 2010). The majority of motif annotations are taken from the Pfam and SMART databases (see Tables 1 and 2; Letunic et al. 2009; Finn et al. 2010). (A) HRD1 ligase complex: This complex is a well-illustrated E3 complex that primarily targets proteins for ERAD-L. OS-9 and XTP3-B recognize aberrant nonglycosylated or glycosylated proteins in the ER lumen. Both associate with the HRD1 complex through SEL1L in a mutually exclusive manner. BiP and GRP94 presumably cooperate to regulate the assembly/disassembly of the HRD1 complex and sequester misfolded proteins to prevent other interactions until retrotranslocation. Derlin family proteins (Derlin 1, 2, or 3) might participate in substrate retrotranslocation from the ER lumen into the cytosol. (Legend continues on following page.) 
Figure 2. (Continued) UBXD8 and UBXD2 bind to p97/VCP through their UBX domain and accelerate the degradation of ERAD substrates. E2 ubiquitin-conjugating enzymes (Ube2j1, Ube2k) mediate substrate ubiquitination (Burr et al. 2011), whereas the p97/VCP hexamer promotes substrate extraction into the cytosol. Ubiquilin-1 is suggested to act as a ubiquitin-proteasome shuttle protein. Other ubiquitin-chain modifiers may also come into play, such as E4 ubiquitin-conjugating enzyme (Ufd2), Ufd2 inhibitor (Ufd3), and deubiquitinases (Ataxin-3, YOD1, VCIP135) (Rumpf and Jentsch 2006). The deglycosylating enzyme PNGase releases Nlinked glycan chains from the glycopeptide (Tanabe et al. 2006). The proteasome then captures the polyubiquitin chains on the substrate through specific subunits (Rpn10/13, Rpt5) and degrades it. (B) RMA1 ligase complex: RMA1/RNF5 associates with Derlin-1 and E2 Ube2j1. BAP31, known to be an ER sorting factor of diverse membrane proteins, interacts with RMA1 as well as components of the Sec61 pore, which suggests that BAP31 might recruit the ERAD complex to the translocon channel to clear newly synthesized misfolded membrane proteins from the channel. In addition, DNAJB12, which contains the cytosolic J-domain, may also participate in the degradation of membrane proteins together with HSP70. All of these factors have been reported to play a role in the degradation of cystic fibrosis trans-membrane conductance regulator (CFTR) and its mutant (CFTR 4508 ) during the early steps of translation (Younger et al. 2006; Morito et al. 2008). (C) Cytosolic E3 ligases: CHIP (carboxyl terminus of Hsp70-interacting protein) possesses a U-box domain, which has a structure similar to the RING-finger domain, and a tetratricopeptide repeat domain (TPR) that interacts with Hsp70 and Hsp90. In contrast to RMA1, CHIP posttranslationally monitors the folding of CFTR or CFTR $\Delta$ 508. Parkin, which is responsible for autosomal recessive juvenile Parkinsonism, targets several proteins, such as O-glycosylated $\alpha$-synuclein, the Pael receptor (Pael-R), Synphilin-1, and Tau. Regarding Pael-R, Parkin and CHIP act together to enhance its ubiquitination and inhibit cell death induced by accumulated unfolded Pael-R. Parkin also works with the E2 proteins Ube2j1 and Ube2g2, which suggests that it is involved in ERAD. The SCF (SKP1-CUL1-F-box protein) is composed of three proteins (Cullin1, Skp1, and RING finger protein $\mathrm{Rbx} 1$ ) and one F-box protein (Fbs1 or Fbs2). Fbs1 and Fbs2 are novel F-box proteins that recognize sugar chains in N-linked glycoproteins and show a chaperone-like activity to prevent their aggregation (Yoshida and Tanaka 2010). (D) gp78 ligase complex: The carboxyl terminus of gp78 is composed of four motifs: RING, CUE, G2BR (Ube2g2 binding region), and VIM (p97/VCP-interacting motif). The gp78 ligase complex usually consists of Ube2g2, Derlin-1, p97/VCP, and UBXD2 or UBXD8. gp78 mediates several ERAD substrates, including T-cell receptor subunits (CD3- $\delta$ and TCR- $\alpha$ ), apolipoprotein B-100, Insig-1, and HMG-CoA reductase. The latter two substrates suggest that gp78 is involved in cholesterol metabolism (Jo and Debose-Boyd 2010). gp78 also cooperates with RMA1 to degrade CFTR $\Delta 508$. SVIP is reported to inhibit the assembly of the gp78 ligase complex (Derlin-1, gp78, and p97), which suggests an inhibitory effect on ERAD (Ballar et al. 2007). (E) TEB4 (Doa10) ligase complex: TEB4 is known to be a mammalian homolog of yeast Doa10. Together with Ube2g2, TEB4 is implicated in the degradation of ER resident type 2 iodothyronine deiodinase (Zavacki et al. 2009). Based on the homology of the yeast Doa10, TEB4 might also interact with Ube2j1 (Ubc6) and UBXD8 (Ubx2). 


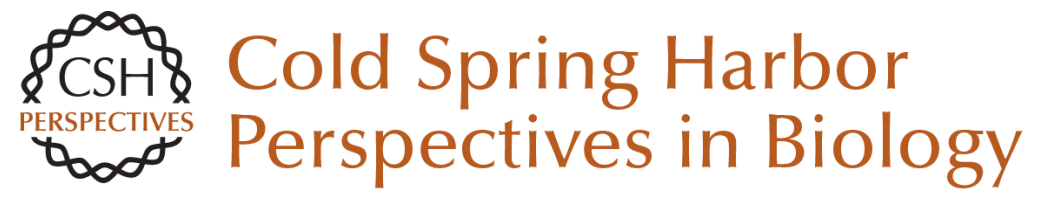

\section{Protein Folding and Quality Control in the ER}

Kazutaka Araki and Kazuhiro Nagata

Cold Spring Harb Perspect Biol 2012; doi: 10.1101/cshperspect.a015438

Subject Collection Protein Homeostasis

Proteome-Scale Mapping of Perturbed

Proteostasis in Living Cells

Isabel Lam, Erinc Hallacli and Vikram Khurana

Pharmacologic Approaches for Adapting

Proteostasis in the Secretory Pathway to

Ameliorate Protein Conformational Diseases Jeffery W. Kelly

Cell-Nonautonomous Regulation of Proteostasis

in Aging and Disease Richard I. Morimoto

The Autophagy Lysosomal Pathway and Neurodegeneration Steven Finkbeiner

Functional Modules of the Proteostasis Network Gopal G. Jayaraj, Mark S. Hipp and F. Ulrich Hartl

Protein Solubility Predictions Using the CamSol Method in the Study of Protein Homeostasis Pietro Sormanni and Michele Vendruscolo

Recognition and Degradation of Mislocalized Proteins in Health and Disease Ramanujan S. Hegde and Eszter Zavodszky

The Nuclear and DNA-Associated Molecular Chaperone Network

Zlata Gvozdenov, Janhavi Kolhe and Brian C. Freeman
The Amyloid Phenomenon and Its Significance in Biology and Medicine

Christopher M. Dobson, Tuomas P.J. Knowles and Michele Vendruscolo

A Chemical Biology Approach to the Chaperome

in Cancer--HSP9O and Beyond

Tony Taldone, Tai Wang, Anna Rodina, et al.

Proteostasis in Viral Infection: Unfolding the Complex Virus-Chaperone Interplay Ranen Aviner and Judith Frydman

The Proteasome and Its Network: Engineering for Adaptability Daniel Finley and Miguel A. Prado

Functional Amyloids Daniel Otzen and Roland Riek

Chaperone Interactions at the Ribosome Elke Deuerling, Martin Gamerdinger and Stefan G. Kreft

Mechanisms of Small Heat Shock Proteins Maria K. Janowska, Hannah E.R. Baughman, Christopher N. Woods, et al.

Structure, Function, and Regulation of the Hsp90 Machinery Maximilian M. Biebl and Johannes Buchner

For additional articles in this collection, see http://cshperspectives.cshlp.org/cgi/collection/

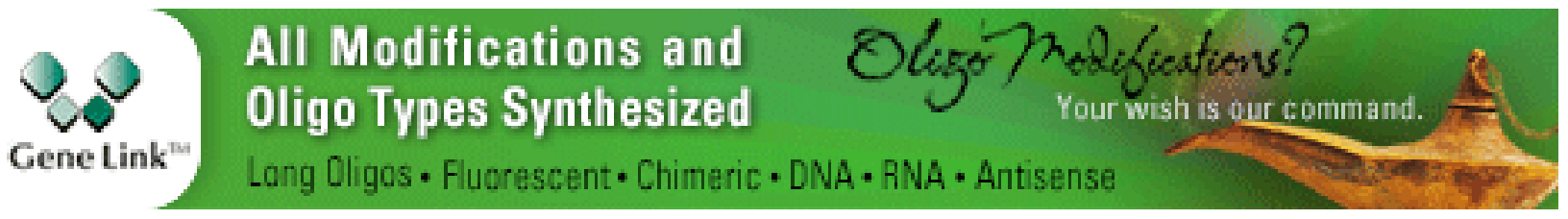


For additional articles in this collection, see http://cshperspectives.cshlp.org/cgi/collection/

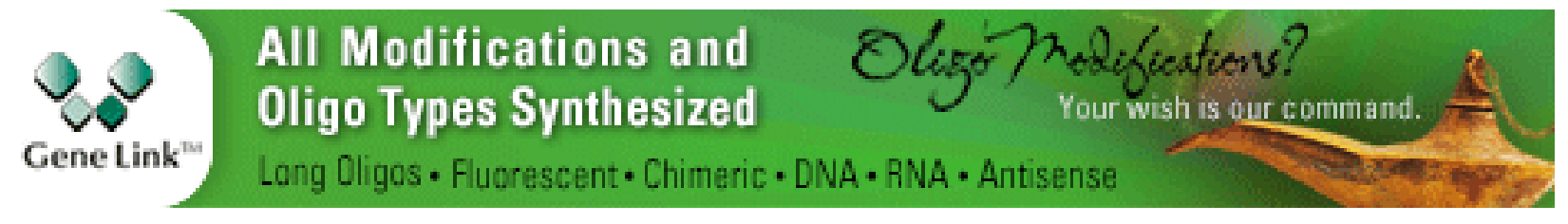

Copyright @ 2012 Cold Spring Harbor Laboratory Press; all rights reserved 